\title{
Zur Ermittelung von Explosionsgrenzen in Gasgemengen;
}

von

Nic. Teclu.

Zahlreiche Versuche zur Bestimmung von Explosionsgrenzen in Gasgemengen führten zur Feststellung eines diesbezüglichen Vorganges, welcher sehr befriedigende Resultate ergab. Hierzu diente eine Vorrichtung aus Glas, deren Einrichtung aus umstehender Zeichnung ersichtlich ist. ${ }^{1}$ ) Er besteht im wesentlichen aus einem gebogenen Eudiometer $A$ und $B^{2}$ ), an dessen Bugstelle sich das rechtwinklig nach unten angesetzte Rohr $J^{3}$ ) befindet. Der VerschluB des Eudiometers, bei den Platinpolen, ist durch den Zweiweghahn $E^{4}$ ) bewerkstelligt, auf welchem, in gleicher Richtung des Eudiometers, die Röhre $C^{5}$ ) angesetzt ist; sie steht an ihrer verjüngten Stelle mit dem Glashahn $G^{6}$ ) in Verbindung und mündet mit der rechtwinklig gebogenen Röhre $L$. Am Fuße der Röhre $C$ ist ferner ein doppelt rechtwinklig gebogenes Rohr mit dem Hahn $F$ angebracht; sie mündet bei $K$. Dieser gegenüber befindet sich ein, nach unten schief gerichtetes, Ansatzrobr $S t^{7}$ ), sowie eine, auf dem $Z_{\text {weiweghahn aufgesetzte }}$

1) Dieser Apparat wird in Wien von W. J. Rohrbecks Nachfolger und in Leipzig von Franz $\mathrm{Hugershoff}$ angefertigt.

2) $A$ ist $20 \mathrm{~cm}, B 85 \mathrm{~cm}$ lang und beide haben die Weite von $14 \mathrm{~mm}$. Die Skale auf $A$ reicht bis $20 \mathrm{~cm}$ und besitzt eine Millimeterteilung; von gleicher Höhe und Beschaffenheit ist die Skale auf $B$ angebracht.

s) Das Ansatzrohr $J$ hat die Länge von $5 \mathrm{~cm}$ und die Weite ron $8 \mathrm{~mm}$.

4) Der Zweiweghahn $E$ besitzt eine Hauptbohrung von $2 \mathrm{~mm}$ Querschnitt und eine Nebenbohrung von $1 \mathrm{~mm}$ Durchmesser

s) Die Röhre $C$ ist $3 \mathrm{~cm}$ weit und $10 \mathrm{~cm}$ lang; sie besitzt eine Centimeter-Skale, ohne Unterabteilungen.

6) Der Glashahn $G$ hat eine Bohrung von $1 \mathrm{~mm}$ Querschnitt.

7) Die Länge des Ansatzrohres $S t$ beträgt $3 \mathrm{~cm}$; der Querschnitt derselben $8 \mathrm{~mm}$. 
Teclu: Zur Ermittelung ron Explosionsgrenzen ete. 213

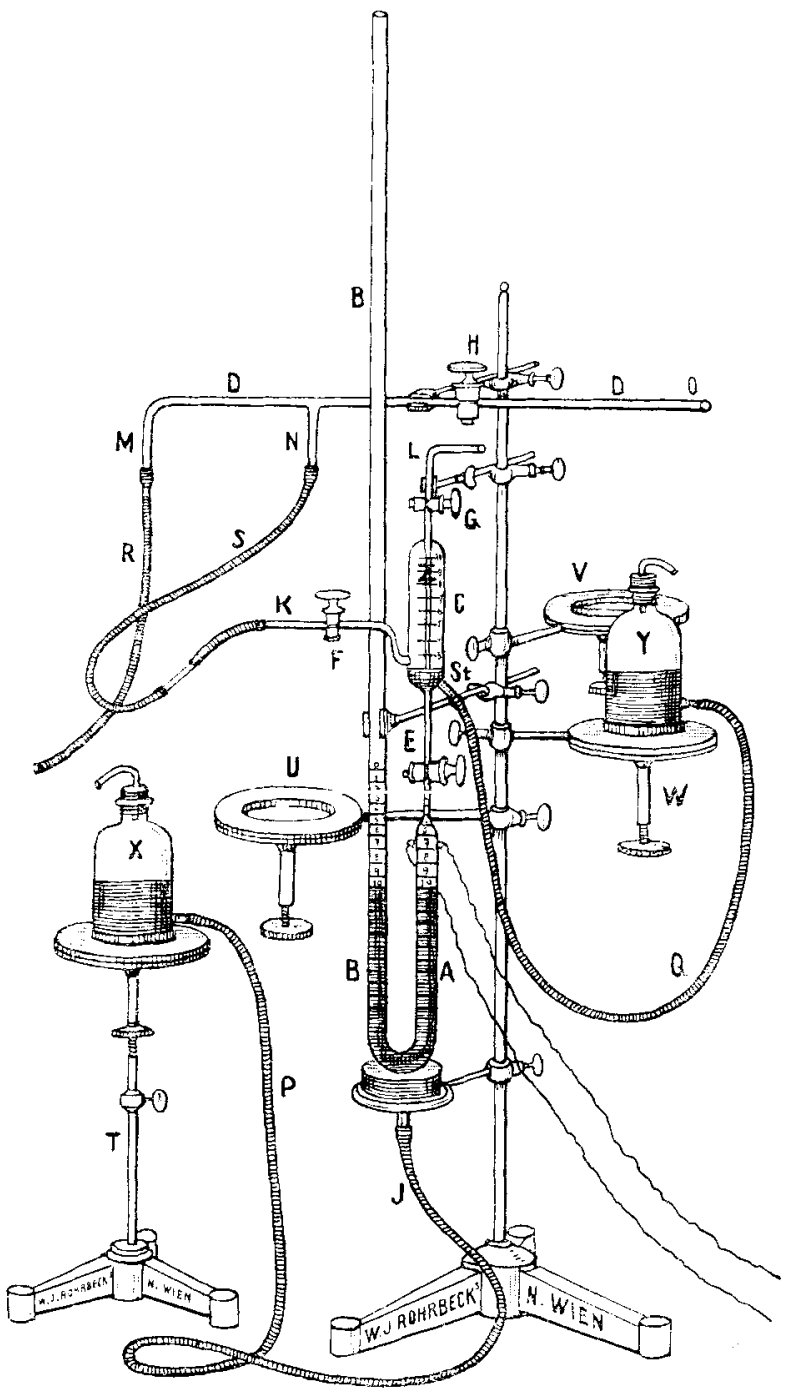

Röhre $Z^{1}$ ), welche im Innern der Röhre $C$, in der Mitte aufsteigt und mit ihrem Ende nahezu bis zum Hahn $G$ reicht. Der Apparat ist in geeigneter Weise an ein eisernes

1) Die Röhre $Z$ ist $9,8 \mathrm{~cm}$ lang und $3 \mathrm{~mm}$ weit; an ihrem obersten Ende verjüngt sie sich bis zum Querschnitt von $1 \mathrm{~mm}$. 
Stativ mit Klemmen derart befestigt, daß die Eudiometerröhre in lotrechter Richtung feststeht, letztere hat, mit Rücksicht auf die Erschütterungen, welche bei den Explosionen stattfinden, an der Bugstelle einen Kautschukpfropf als Unterlage, durch dessen Bohrung die Röhr^ In durchgesteckt ist.

Andere Teile des Apparates, die ebenfalls durch entsprechende Klemmvorrichtungen an das Stativ befestigt sind, bilden, zunächst die mit dem Hahn $H$ versehene Röhre $D^{2}$ ), und weiter die drei Stativ-Ringe samt den entsprechenden Einsätzen aus Messing $U, V$ und $W$. Letztere bestehen aus Messingscheiben von der Größe der Ringe und sind mit einem schmalen Reif eingefaßt; sie können mit Hilfe einer Schraube höher und niederer gestellt werden. In nächster Nähe dieses Apparates steht ein Stativtisch $T$, dessen Platte den früher erwähnten Einsätzen der Stativ-Ringe gleicht und ebenfalls mit Hilfe einer Schraube in kleinen Abständen, nach oben oder nach unten, verstellt werden kann.

An den Rohrenden des Apparates $J, S t, M$ und $N$ sind schwarze Kautschukschläuche ${ }^{3}$ ) angebracht, von denen jene, der Röhren $J$ und $S t$ mit den tubulierten Flaschen $\left.{ }^{4}\right) X$ und $Y$ in Verbindung stehen, welche Quecksilber enthalten. Diese Schläuche werden zum Durchleiten des letzteren benutzt; die anderen, mit den Röhren $M$ und $N$ verbundenen, dienen zum Durchleiten von Gasen.

Man kann durch Heben und Senken der Flaschen das

5) Die Länge dieser Röhre beträgt $5 \mathrm{~cm}$; ihre Weite $8 \mathrm{~mm}$.

2) Die Röhre hat eine horizontale Lage; sie ist $30 \mathrm{~cm}$ lang und $1,2 \mathrm{~cm}$ weit. In der Mitte trägt sie den Hahn $H$, dessen Bohrung im Querschnitt $5 \mathrm{~mm}$ beträgt. Das eine, nach unten gebogene Rohrende $M$, dient als Schlauchansatz. Zwischen diesem und dem Hahne $H$ befindet sich, rechtwinklig angesetzt, noch ein Schlauchansatzrohr $N$, während die Röhre $D$, auf der anderen Seite des Hahnes, ohne Unterbrechung mit dem offenen Ende $O$ mündet.

3) Der an dem Rohrende $S t$ befestigte Kautschukschlauch, aus Patentgummi I hergestellt, hat die Länge von $55 \mathrm{~cm}$ und den Querschnitt von $5 \mathrm{~mm}$; auch der an der Röhre $J$ befestigte Schlauch ist von derselben Qualität und ist $110 \mathrm{~cm}$ lang, während die mit den Rohrenden $M$ und $N$ verbundenen Schläuche von gewöhnliche Qualität sind; ersterer ist $90 \mathrm{~cm}$, letzterer $50 \mathrm{~cm}$ lang und beide etwa $\mathrm{mm}$ weit.

4) Der Fassungsraum der Flaschen beträgt je 0,3 Liter. 
Teclu: Zur Ermittelung von Explosionsgrenzen etc. 215

Quecksilber im Eudiometer, oder in der Röhre $C$ höher und niederer stellen und, wenn die Unterlage der Flaschen vorher auf eine bestimmte Höhe eingestellt wurde, den Höhenstand des Quecksilbers in den Röhren feststellten. Endlich verbindet der Kautschukschlauch $R$ die Röhre $D$, und der Kautschukschlauch $S$, der an das Rohrende $N$ angesetzt wird, das Rohrende $K$ und somit das Gefäß $C$.

Die ausgeführten Untersuchungen beziehen sich zunächst auf Bestimmungen von Explosionsgrenzen in Luftgemengen. Zur Aufnahme eines bestimmten Luftvolumens dient das Gefäß $C$, welches ganz oder zum Teil angefült werden kann, je nach der Einstellung des Ringeinsatzes $\boldsymbol{W}$, auf welchen die Flasche $F$ gestellt wird. Reicht das Quecksilber im Gefäße $C$ bis zum höchsten Teilstriche 1 der Skale, dann faßt das Gefäb samt dem Inhalt der Röhre $\boldsymbol{Z}$ 6,2 cem Luft,

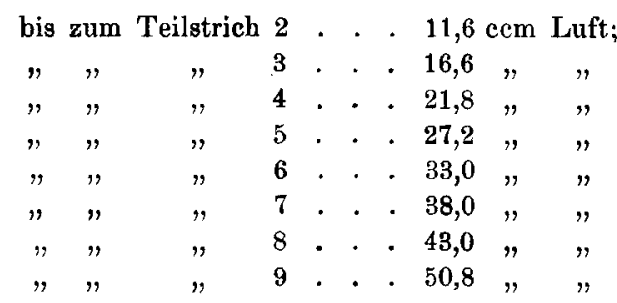

Der andere gasförmige Bestandteil des Gemenges wird in das Eudiometer eingeführt und sein Volum von der Skale abgelesen; die kleinste direkt bestimmbare Menge desselben in diesem Apparate beträgt $1,3 \mathrm{ccm}$, die größte $21,62 \mathrm{ccm}$. Dadurch, daB sowohl im Eudiometer, als auch im GefäBe $C$ verschiedene Mengen des betreffenden Gases abgesondert werden können, insbesondere aber deshalb, weil nicht nur der ganze Inhalt des Gefäßes $C$ von $50,8 \mathrm{ccm}$, sondern auch dieselbe Menge, oder Teile desselben, wiederholt für ein und dieselbe Bestimmung hintereinander in Verwendung kommen können, ist es möglich, das Mischungsverhältnis des explosiven Gemenges nach Belieben zu regeln.

Zur Erläuterung des Vorganges bef solchen Bestimmungen möge beispielsweise ein Gemenge von Wasserstoff und Luft dienen. $Z$ u diesem $Z_{\text {wecke }}$ wird zunächst die Flasche $X$ auf den Stativ-Tisch $T$ gestellt und dessen Höhenstellung, bei 
offenen Hähnen des Eudiometers, so geregelt, daB das Quecksilber in denselben bis zum gewünschten Teilstriche reicht, etwa bis zu jenem von 10 , entsprechend $8,92 \mathrm{ccm}$. Nachher wird die Flasche $X$ auf den Ringeinsatz $U$ gestellt, deren Höhenstand nicht nur die Füllung des Eudiometers $A$ verursacht, sondern auch die der Hauptbohrung des Zweiweghahns. Man wartet, bis das Quecksilher zur Rube gekommen ist, und dreht dann den Hahn um $90^{\circ}$ nach rechts, wodurch seine Hauptbohrung geschlossen, seine Nebenbohrung geöffnet wird. Anderseits bringt man, durch die Stellung der Flasche $Y$ auf den Ringeinsatz $W$, den Höhenstand des Quecksilbers, in dem GefäBe $C$, auf den Teilstrich 9, entsprechend der Luftmenge von $50,8 \mathrm{ccm}$. Nun leitet man Wasserstoff aus einem Kippschen Apparate, mit Hilfe von Zink und Schwefelsäure dargestellt, und mittelst konzentrierter Schwefelsäure getrocknet, durch den Kautschukschlauch $R$ in die Röhre $D$. Von hier gelangt dieser, da der Hahn $H$ geschlossen ist, in den Kautschukschlauch $S$, ferner in die Röhre $K$, durch den Hahn $F$ in das GefäB $C$ und von da, durch die Hähne $E$ und $G$ ins Freie. Nach wenigen Minuten wird hierdurch die Luft aus dem Gefäße $C$ verdrängt und jene, die sich in der Röhre $Z$ befindet, entweicht beim Schließen des Hahnes $G$ durch die Nebenbohrung des Zweighahnes. Man öffnet ${ }^{1}$ ) nun den $Z$ weighahn, stellt von dem Ringeinsatz $U$ die Flasche $X$ auf den Stativ-Tisch $T$, wodurch das Quecksilber in beiden Teilen des Eudiometers sinkt. Dies geschieht nicht gleichmäßig, und es kann letzteres auch sein Gleichgewicht in beiden Röhren nicht erlangen, da der Überdruck des Wasserstoffs einseitig zur Wirkung kommt; erst nach dem Offnen des Hahnes $H$ erlangt das Quecksilber in beiden Teilen des Eudiometers eine gleiche Höhe. Wird nun der Zweiweghahn geschlossen ${ }^{2}$ ), so ist damit das ursprünglich beabsichtigte Wasserstoff -Volum von $8,92 \mathrm{ccm}$ abgesondert und muß nun mit $\mathrm{Luft}^{3}$ ) gemischt werden. Um

1) Der Zweighahn kommuniziert in diesem Falle mittels seiner Hauptbohrung mit dem Eudiometer, wobei die Nebenbohrung des Hahnes geschlossen ist.

$\left.{ }^{2}\right)$ Hierdurch wird die weitere Bohrung des Zweiweghahnes abgesperrt, die engere geöffnet.

3) Da die Explosionsempfindlichkeit des Gasgemisches, durch den Feuchtigkeitsgehalt der Luft, in nicht geringem Grade beeinflußt wird 
dies zu bewirken, beseitigt man den Wasserstoff aus dem GefäBe $C$, indem durch letzteres Luft geleitet wird. ${ }^{1}$ ) Man entfernt den Kautschuksehlauch $S$, öffnet den Hahn $G$ und führt bei $L$ die Luft in den Apparat ein. Diese strömt durch das Gefäß $C$ und das Rohr $Z$, wenn der Hahn $F$ offen ist, beim SchlieBen des letzteren nur durch das Rohr $Z$, und verläßt den Apparat durch die Nebenbohrung des Zweighahnes. In kürzester Zeit ist aus dem Gefäße $C$ und der Röhre $Z$ aller Wasserstoff verdrängt; es wird der Hahn $F$ und der Hahn $G$ geschlossen, womit $50,8 \mathrm{ccm}$ Luft abgesondert ${ }^{2}$ ) werden. Man hat nun die $50,8 \mathrm{ccm}$ luft mit den $8,92 \mathrm{ccm}$ Wasserstoff zu mischen, was durch Öffnen des Zweiweghahnes und Heben der Flasche $X$ bewirkt werden kann. Es steigt hierdurch das Quecksilber in beiden Teilen des Eudiometers, der Wasserstoff gelangt allmählich ${ }^{3}$ ) in die Röhre $Z$, von da in das GefäB $C$ und mengt sich hier mit der Luft. ${ }^{4}$ ) Hat das Quecksilber die Spitze der Röhre $Z$ erreicht, dann senkt man die Flasche so lange, bis der ursprüngliche Höhenstand des Quecksilbers wieder erreicht wird, wiederholt diesen Vorgang noch zweimal, wodurch die gleichförmige Mischung der Gase bewerkstelligt ist. ${ }^{5}$ ) Von diesem Gemenge sondert man, durch SchlieBen des Zweiweghahnes ${ }^{6}$ ), einen Teil im Eudiometer

und yollständig trockene Luft, nur sebr umständlich zu beschaffen ist, wurde die Luft, damit sie, wenn auch noch geringen, so doch möglichst gleichen Feuchtigkeitsgehalt besitze, in allen Fällen über konzentrierte Schwefelsăure geleitet, die vermittelst Glasperlen in Glastürmen zur Wirksamkeit gelangte.

1) Dies kann mit Hilfe eives Gasometers geschehen; bei den vorliegenden Untersuchungen wurde ein Trommelgebläse verwendet.

2) Es wird hierdurch die Hauptbohrung des Zweiweghahnes in lotrechte Lage gebracht, wodurch die Gase ineinander strömen können; die Nebenbohrung des Zweiweghahnes ist hierbei geschlossen.

3) Die Bewegungen mit der Flasche dïrfen nicht zu rasch erfolgen, da sonst die Hähne unverläßlich werden.

4) Während das steigende Quecksilber den Wasserstoff durch die Röhre $Z$ in das Gefäß $C$ preßt, sinkt das in diesem Gefäße vorhandene Quecksilber und wird in die Flasche $Y$ gehoben.

$\left.{ }^{5}\right)$ Bei richtigem Gange der Untersuchung muB sich das Quecksilber auf denselben Teilstrich, wie vorher, einstellen.

${ }^{6}$ ) Er wird um $90^{\circ}$ gedreht. Hierdurch schliebt sich seine Hauptbohrung und öffnet sich seine Nebenbohrung.

Journal f. prakt. Chemie [2] Bd. 75. 
$\left.a b^{1}\right)$ und läBt durch diesen, mit Zuhilfenahme eines Induktionsapparates, den Funken überspringen. ${ }^{2}$ ) Im vorliegenden Falle, da das Gasgemenge aus $8,92 \mathrm{ccm}$ Wasserstoff und $50,8 \mathrm{ccm}$ Luft besteht und demnach 14,94\% Wasserstoff enthält, bewirkt der Funke eine Explosion. Das Quecksilber im Eudiometerrohr $B$ wird plötzlich niedergepreBt und im Eudiometerrohr $A$ emporgeschleudert. ${ }^{3}$ ) Nachdem es zur Ruhe gekommen ist, wird die eingetretene Kontraktion und die Bildung von Wasser ersichtlich. Um einen weiteren Versuch ausführen zu können, muB das Wasser beseitigt werden, was mittels Durchleiten von Luft ziemlich rasch $\mathrm{zu}$ erreichen ist. Hierzu wird der Zweiweghahn geöffnet, die Flasche $X$ so tief gestellt, daB das Quecksilber aus dem Eudiometer vollständig durch die Röhre $J$ abfließen kann. Dann verbindet man den Kautschukschlauch von einer Saugpumpe mit der Röhre $L$, öffnet den Hahn $G$ und schlieBt den Hahn $F$. Eine weitere Erscheinung bei diesen Untersuchungen ist das Auftreten von Bläschen an den Wandungen des Eudiometers. Diese Luftbläschen beseitigt man aus dem Eudiometerrohr $B$ durch entsprechendes Heben und Senken der Flasche $X$, bezw. des Quecksilbers, dagegen jene aus der Eudiometerröhre $A$ durch Neigen des Apparates, wobei, wenn der Wert der Kontraktion zu bestimmen ist, der Zweiweghahn nicht geöffnet werden darf.

1) Man kann diese Versuche auch so ausführen, dab das abgesonderte Explosionsgemenge bei allen Bestimmungen das gleiche bleibt. Von vornherein wird in solchen Fällen ein größeres Volumen des Explosionsgemenges abgesondert und der vorhandene Überschuß aus dem Apparat entfernt. Um dies zu bewerkstelligen, muß die Einstellungsvorrichtung der Flasche $\boldsymbol{X}$ für das EinheitsmaB vorgerichtet sein und die Flasche für kurze Zeit auf diese Unterlage übertragen werden. Das steigende Quecksilber verdrängt dann einen Teil des Explosionggemisches durch die Nebenbohrung des Zweiweghabnes, wenn dieser um $90^{\circ}$ nach links gedreht wird.

2) $\mathrm{Zu}$ den vorliegenden Untersuchungen, welche mehrere Monate in Anspruch nahmen, schien es vorteilhafter, den Gassenstrom zu ververwenden und den Rheostaten von Max Kohl in Chemnitz einzuschalten.

s) Die Glasstärke des Eudiometers ist die übliche und hinlänglich widerstandsfähig; bei den zahlreichen Versuchen wurde das Eudiometer nie beschädigt. 
Teclu: Zur Ermittelung von Explosionsgrenzen etc. 219

Nach diesem Vorgange wurde zunächst die untere Explosionsgrenze des Wasserstoffs bestimmt. Die hierbei erhaltenen Werte waren folgende:

\begin{tabular}{|c|c|c|c|c|c|}
\hline \multicolumn{2}{|c|}{ Wasserstoff } & \multicolumn{2}{|c|}{ Luft } & \multirow{2}{*}{$\frac{\text { Wasserstoff }}{\begin{array}{c}\text { Prozent- } \\
\text { gehalt }\end{array}}$} & \multirow{2}{*}{$\begin{array}{l}\text { Gemenge } \\
\text { Funken- } \\
\text { wirkung }\end{array}$} \\
\hline Teilstriche & $\mathrm{cem}$ & Teilstriche & $\mathrm{ccm}$ & & \\
\hline 6,0 & 3,84 & 9,0 & 50,8 & 7,03 & keine \\
\hline 7,0 & 5,11 & , & , & 9,14 & ," \\
\hline 7,2 & 5,36 & $"$ & , & 9,73 & $"$ \\
\hline 7,4 & 5,62 & $"$ & " & 9,96 & Explosion \\
\hline
\end{tabular}

Aus diesen Versuchsergebnissen $\left.{ }^{1}\right)$ resultiert, daB die untere Explosionsgrenze des Wasserstoffs zwischen $9,73 \%$ und $9,96 \%$ liegt.

Für die obere Explosionsgrenze des Wasserstoffs wurden folgende Zahlen erhalten:

\begin{tabular}{c|c||c|c||c|c}
\hline \hline \multicolumn{2}{c||}{ Wasserstoff } & \multicolumn{2}{c|}{ Luft } & Wasserstoff & Gemenge \\
\hline Teilstriche & ccm & Teilstriche & ccm & $\begin{array}{c}\text { Prozent- } \\
\text { gehalt }\end{array}$ & $\begin{array}{c}\text { Funken- } \\
\text { wirkung }\end{array}$ \\
\hline \hline 13,0 & 12,73 & 1,0 & 6,2 & 67,25 & keine \\
12,5 & 12,095 & $"$ & $"$ & 66,11 & $"$ \\
12,0 & 11,46 & $"$ & $"$ & 64,89 & $"$ \\
11,5 & 10,83 & $"$ & $"$ & 63,58 &, \\
11,2 & 10,44 & $"$ & $"$ & 62,75 & Explosion
\end{tabular}

aus welchen sich der Grenzwert zwischen $62,75 \%$ und $63,58 \%$ ergibt.

Um die Explosionsgrenzen des Leuchtgases ${ }^{2}$ ) aufzufinden, wurde dieses unmittelbar aus der Gasleitung entnommen und mittels des Kautschukschlauches $R$ durch die Röhre $K$ in den

1) Bei allen diesen Bestimmungen, die bei gewöhnlicher Zimmertemperatur ausgeführt wurden, ist jedesmal sowohl die Temperatur, als auch der Druck beobachtet worden. Ihre Angabe fehlt, weil sie für diese Untersuchungen kaum zu berücksichtigen sind.

$\left.{ }^{2}\right)$ Das zu den Untersuchungen verwendete Leuchtgas stammt aus den städtischen Gaswerken. 
220 Teclu: Zur Ermittelung von Explosionsgrenzen etc.

Apparat geleitet. Die Versuchsresultate zur Feststellung der unteren Explosionsgrenze sind folgende:

\begin{tabular}{|c|c|c|c|c|c|}
\hline \multicolumn{2}{|c|}{ Leuchtgas } & \multicolumn{2}{|c|}{ Luft } & \multirow{2}{*}{$\begin{array}{c}\text { Leuchtgas } \\
\begin{array}{c}\text { Prozent- } \\
\text { gehalt }\end{array}\end{array}$} & \multirow{2}{*}{$\begin{array}{l}\text { Gemenge } \\
\text { Funken- } \\
\text { wirkung }\end{array}$} \\
\hline Teilstriche & ecm & Teilstriche & $\mathrm{ccm}$ & & \\
\hline 6,0 & 3,84 & 9,0 & 50,8 & 7,03 & Explosion \\
\hline 5,8 & 3,59 & $"$ & , & 6,56 & " \\
\hline 5,6 & 3,33 & $"$ &, & 6,16 & $"$ \\
\hline 5,4 & 3,08 & $"$ & $"$ & 5,71 & $n$ \\
\hline 5,2 & 2,82 & " & $"$ & 5,27 & $"$ \\
\hline 5,0 & 2,57 & $"$ & $"$ & 4,84 & $"$ \\
\hline 4,8 & 2,32 & $n$ & $"$ & 4,30 & keine \\
\hline
\end{tabular}

aus welchen folgt, daB diese auf den Wert zwischen $4,36 \%$ und $4,82 \%$ weisen.

Die obere Explosionsgrenze des Leuchtgases ergibt sich aus folgenden Versuchen:

\begin{tabular}{|c|c|c|c|c|c|}
\hline \multicolumn{2}{|c|}{ Leuchtgas } & \multicolumn{2}{|c|}{ Luft } & \multirow{2}{*}{$\begin{array}{c}\text { Leuchtgas } \\
\begin{array}{c}\text { Prozent- } \\
\text { gehalt }\end{array}\end{array}$} & \multirow{2}{*}{$\begin{array}{l}\text { Gemenge } \\
\text { Funken- } \\
\text { wirkung }\end{array}$} \\
\hline Teilstriche & $\mathrm{cem}$ & Teilstriche & $\mathrm{ccm}$ & & \\
\hline 10,0 & 8,92 & 5,0 & 27,2 & $\mathbf{2 4 , 6 9}$ & keine \\
\hline 9,8 & 8,67 & 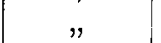 & , & 24,16 & $"$ \\
\hline 9,6 & 8,41 & , & , & 23,63 & 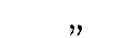 \\
\hline 9,5 & 8,29 & $"$ & , & $23,3 \tilde{5}$ & Explosion \\
\hline
\end{tabular}

und liegt zwișchen $23,35 \%$ bis $23,63 \%$

Die Untersuchung des Sumpfgases ${ }^{1}$ ) auf seine untere Explosionsgrenze weist folgende Werte auf:

1) Das Sumpfgas wurde aus Aluminiumcarbid (E. Merck, Darmstadt) dargestellt. In einen Kochkolben von 1,5 Liter Inhalt bringt man $150 \mathrm{~g}$ Carbid, das man mit auf $40^{\circ}$ erwärmtem Wasser übergiebt. Der Kolben trägt einen durchbohrten Pfropf, durch den luftdicht ein Glasrohr geht, welches mit einem Kautschukschlauch verbunden ist. Das Gas entwickelt sich anfangs langsam, verdrängt die Luft aus dem Kolben, als auch aus der Röhre, sowie aus dem Schlauche und gelangt, durch konzentrierte Schwefelsäure geleitet, in den Gasometer, von wo es für die Versuche entnommen wird. Da bei der Entwicklung dieses Gases die Reaktion immer lebhafter wird, empfiehlt es sich, den Kolben von Zeit zu Zeit in kaltes Wasser zu tauchen. 
T'eclu: Zur Ermittelung von Explosionsgrenzen etc. 221

\begin{tabular}{|c|c|c|c|c|c|}
\hline \multicolumn{2}{|c|}{ Sumpfgas } & \multicolumn{2}{|c|}{ Luft } & \multirow{2}{*}{$\begin{array}{c}\text { Sumpfgas } \\
\text { Prozent- } \\
\text { gehalt }\end{array}$} & \multirow{2}{*}{$\begin{array}{l}\text { Gemenge } \\
\text { Funken- } \\
\text { wirkung }\end{array}$} \\
\hline Teilstriche & cem & Teilstriche & $\mathrm{cem}$ & & \\
\hline 6,0 & 3,84 & 9,0 & 50,8 & 7,03 & Explosion \\
\hline 5,5 & 3,21 & , & , & 5,93 & " \\
\hline 5,0 & 2,75 & $"$ & " & 4,82 & ", \\
\hline 4,7 & 2,19 & " & $n$ & 4,13 & , \\
\hline 4,5 & 1,94 & $"$ & 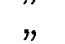 & 3,67 & $"$ \\
\hline 4,3 & 1,67 & $"$ & $"$ & 3,20 & keine \\
\hline
\end{tabular}

welche Werte der Explosionsgrenze zwischen $3,20-3,67 \%$ entsprechen.

Die obere Explosionsgrenze des Sumpfgases ergibt sich aus folgenden Bestimmungen:

\begin{tabular}{|c|c|c|c|c|c|}
\hline \multicolumn{2}{|c|}{ Sumpfgas } & \multicolumn{2}{|c|}{ Luft } & \multirow{2}{*}{$\begin{array}{c}\text { Sumpfgas } \\
\text { Prozent: } \\
\text { gehalt }\end{array}$} & \multirow{2}{*}{$\begin{array}{c}\text { Gemenge } \\
\text { Funken- } \\
\text { wirkung }\end{array}$} \\
\hline Teilstriche & $\mathrm{ccm}$ & Teilstriche & $\mathrm{ccm}$ & & \\
\hline 8,0 & 6,38 & 9,0 & 50,8 & 11,16 & keine \\
\hline 7,0 & 5,11 & , & $"$ & 9,14 & " \\
\hline 6,8 & 4,86 & " & $"$ & 8,71 & ", \\
\hline 6,6 & 4,60 & $"$ & $"$ & 8,31 & $n$ \\
\hline 6,4 & 4,35 & $"$ & $"$ & 7,88 & , \\
\hline 6,2 & 4,09 & $"$ & $"$ & 7,46 & Explosion \\
\hline
\end{tabular}

aus welchen sich für die Explosionsgrenze der Wert von $7,46-7,88 \%$ ergibt.

Bei der Ermittelung der Explosionsgrenzen des Acetylens ${ }^{1}$ ) zeigen sich eine Reihe bemerkenswerter Erscheinungen. $\mathrm{Zu}$ nächst vergrößert sich, wenn geringere Mengen von Acetylen in Anwendung kommen, das Gasvolum nach der Explosion nicht, es tritt vielmehr eine Kontraktion ein. ${ }^{2}$ ) Diese beginnt

1) Das Acetylen wurde aus dem im Handel (W. J. Rohrbecks Nacbfolger in Wien) vorkommenden Calciumcarbid dargestellt. Es diente hierzu eine in kaltem Wasser kühl gehaltene Tropfvorrichtung, und ein Apparat, bestehend aus zwei, je 15 Liter fassenden, vermittelst ibrer Tuben und einen Kautschukschlauchs kommunizierenden Glasflaschen. Zu den Versuchen wurde über Chlorealcium getrocknetes Acetylen verwendet.

$\left.{ }^{2}\right)$ Diese Erscheinung dürfte wohl auf den Umstand zurückzuführen sein, daB das Explosionsgemenge verhältnismäBig klein ist und das entstehende Wasser das gleichzeitig gebildete Kohlendioxyd zum teil absor- 
mit der unteren Explosionsgrenze des Acetylens und hält an bis zu einem Gehalt dieses Gases zwischen 6,38\% und 7,03\% Dann folgt bei Explosionen mit mehr Acetylengehalt eine Vergrößerung des rückständigen Volums, welche ihre Grenze zwischen $7,03 \%$ und $41,92 \%$ hat. Zwischen $41,92 \%$ und $49,79 \%$ Acetylengehalt konnte weder eine Kontraktion, noch eine Expansion mit Sicherheit beobachtet werden. Endlich tritt bei $49,79 \%$ Acetylen bis zur oberen Explosionsgrenze dieses Gases stets schwache Kontraktion ein. Kennzeichnend ist ferner, daB der größte Teil der Explosionserscheinungen von RuBabscheidung und zum geringen Teil von der Bildung trockener Destillationsprodukte begleitet wird. ${ }^{1}$ ) Die Grenze der RuBbilaung liegt zwischen $9,33 \%$ und $9,82 \%$ Acetylen und reicht bis zur oberen Explosionsgrenze dieses Kohlenwasserstoffs.

Die untere Explosionsgrenze des Acetylens wird aus folgenden Versuchsergebnissen ersichtlich:

\begin{tabular}{|c|c|c|c|c|c|c|c|}
\hline & & \multicolumn{4}{|c|}{ Luft. ${ }^{2}$ ) } & & \\
\hline \multicolumn{2}{|c|}{ Acetylen } & \multicolumn{2}{|c|}{ Erste Füllung } & \multicolumn{2}{|c|}{ Zweite Füllung } & \multirow{2}{*}{$\frac{\text { Acetylen }}{\begin{array}{c}\text { Prozent- } \\
\text { gehalt }\end{array}}$} & \multirow{2}{*}{$\begin{array}{l}\text { Gemenge } \\
\text { Funken- } \\
\text { wirkung }\end{array}$} \\
\hline $\begin{array}{c}\text { Teil- } \\
\text { striche }\end{array}$ & $\mathrm{ccm}$ & $\begin{array}{l}\text { Teil- } \\
\text { striche }\end{array}$ & eem & $\begin{array}{c}\text { Teil- } \\
\text { striche }\end{array}$ & ecm & & \\
\hline 6,0 & 3,84 & 9,0 & 50,8 & 1,0 & 6,2 & 2,67 & Explosion \\
\hline 5,5 & 3,21 & $"$ & $"$ & $"$ & $"$ & 2,08 & $"$ \\
\hline 5,3 & 2,95 & $"$ & $"$ & $"$ & $"$ & 1,77 & , \\
\hline 5,1 & 2,697 & $"$ & $"$ & $"$ & $"$ & 1,53 & keine \\
\hline
\end{tabular}

sie liegt zwischen $1,53 \%$ und $1,77 \%$.

biert. Das weitere Verhalten des Gasgemenges nach der Explosion, hinsichtlich seiner Expansion oder Kontraktion, wird durch die teilweise Abscheidung des Kohlenstoffs verursacht, wodurch einerseits weniger Kohlenstoff und verhältnismäfig mehr Wasserstoff zurVerbrennung kommt.

1) Aus diesem Grunde ist es sehr umständlich, die obere Explosionsgrenze des Acetylens zu ermitteln; es muß nach jeder Bestimmung das Eudiometer gereinigt werden, meistens gelingt dies durch Auswaschen mit Schwefelammonium, oft muB zu diesen Zwecken auch noch konzentrierte Schwefelsaure in Anwendung kommen.

2) Die Berechnung des Prozentgehaltes an Acetylen im Explosionsgemenge, wenn beispielsweise $3,84 \mathrm{ccm}$ Acetylen mit mehr Luft gemengt wird, als das Gefäb $C$ Luft faBt, und zwar zuerst mit 50,8 ccm und dann 
Teclu: Zur Ermittelung von Explosionsgrenzen etc. 223

Die obere Explosionsgrenze des Acetylens ergibt sich aus folgenden Daten:

\begin{tabular}{c|c||c|c|c|c}
\hline \multicolumn{2}{c|}{ Acetylen } & \multicolumn{2}{c|}{ Luft } & Acetylen & Gemenge \\
\hline Teilstriche & ccm & Teilstriche & ccm & $\begin{array}{c}\text { Prozent- } \\
\text { gehalt }\end{array}$ & $\begin{array}{c}\text { Funken- } \\
\text { wirkung }\end{array}$ \\
\hline \hline 10,5 & 9,56 & 1,0 & 6,2 & 60,65 & keine \\
10,0 & 8,92 &, & $n$ & 58,99 & $"$ \\
9,9 & 8,79 & $n$ & $n$ & 58,65 & $"$ \\
9,7 & 8,54 & $n$ & $n$ & 57,95 & Explosion
\end{tabular}

und entspricht dem Wert von $57,95 \%$ bis $58,65 \%$.

Die folgende Zusammenstellung der durchgeführten Untersuchungen gestattet die Explosionsgrenzen der verschiedenen Gasgemenge miteinander zu vergleichen:

\begin{tabular}{l|c|c}
\hline \hline \multicolumn{1}{c|}{ Gasart } & $\begin{array}{c}\text { untere } \\
\text { Explosionsgrenze in \% }\end{array}$ & $\begin{array}{c}\text { obere } \\
\text { Explosionsgrenze in } \%_{0}\end{array}$ \\
\hline \hline Wasserstoff & $9,73-9,96$ & $62,75-63,58$ \\
Leuchtgas & $4,36-4,82$ & $23,35-23,63$ \\
Sumpfgas & $3,20-3,67$ & $7,46-7,88$ \\
Acetylen & $1,53-1,77$ & $57,95-58,65$
\end{tabular}

Aus dieser Tabelle ist ersichtlich, daß bezüglich der Explosionsempfindlichkeit das Acetylen obenan steht, das Sumpfgas um 2,1, das Leuchtgas um 2,8 und der Wasserstoff um 6,4 mal weniger reaktionsfähig ist, während bezüglich des Explosionsgebietes dieser Gase das Sumpfgas den kleinsten Umfang besitzt und dieses vom Leuchtgas um 4,21, vom Wasserstoff um 12,7 und rom Acetylen um 13,5 mal ubertroffen wird.

Wien, Chemisches Laboratorium der Wiener HandelsAkademie, im Januar 1907.

mit $6,2 \mathrm{ccm}$, ergibt sich in folgender Weise: wenn $3,84 \mathrm{ccm}$ Acetylen mit 50,8 ccm Luft gemengt werden, so befinden sicb nach der Mengung in dem Raume, der $3,84 \mathrm{ccm}$ faßt, $0,269 \mathrm{ccm}$ Acetylen. Diese Menge findet man aus der Proportion: $(3,84+50,8): 3,84=3.84: x$, oder 54,64: $3,84=3,84: x$, mithin ist $x=0,269$. Das Übrige ist: $3,84-0,269=$ $3,571 \mathrm{ccm}$ Luft. Dazu kommt die nachträglich beigemengte Luft von $6,2 \mathrm{ccm}$. Das nun durch Mischung entstandene Explosionsgemenge besteht demnach aus $0,269 \mathrm{ccm}$ (Acetylen) $+3,571 \mathrm{ccm}$ (Luft) $+6,2 \mathrm{ccm}$ (Luft) $=10,04 \mathrm{ccm}$, in welchem 2,67\% Acetylen enthalten sind, welche sich aus der Proportion: 10,04:0,269 =100:x ergeben. 